\title{
A Novel Substrate Radiotracer for Molecular Imaging of SIRT2 Expression and Activity with Positron Emission Tomography
}

\author{
Robin E. Bonomi, ${ }^{1}$ Maxwell Laws, ${ }^{1}$ Vadim Popov, ${ }^{1}$ Swatabdi Kamal, ${ }^{1}$ \\ Shreya Potukutchi, ${ }^{1}$ Aleksandr Shavrin, ${ }^{1}$ Xin Lu, ${ }^{2}$ Nashaat Turkman, ${ }^{1}$ Ren-Shyan Liu, ${ }^{3}$ \\ Thomas Mangner, ${ }^{2}$ Juri G. Gelovani ${ }^{1}$ \\ ${ }^{1}$ Karmanos Cancer Institute, Wayne State University, Detroit, MI, 48202, USA \\ ${ }^{2}$ PET Center, Wayne State University, Detroit, MI, 48202, USA \\ ${ }^{3}$ National Yang-Ming University School of Medicine, Taipei, Taiwan
}

\begin{abstract}
Purpose: The purpose of this study was to develop a SIRT2-specific substrate-type radiotracer for non-invasive PET imaging of epigenetic regulatory processes mediated by SIRT2 in normal and disease tissues.

Procedures: A library of compounds containing tert-butyloxycarbonyl-lysineaminomethylcoumarin backbone was derivatized with fluoroalkyl chains 3-16 carbons in length. SIRT2 most efficiently cleaved the myristoyl, followed by 12-fluorododecanoic and 10fluorodecanoic groups $\left(K_{\text {cat }} / K_{\mathrm{m}} 716.5 \pm 72.8,615.4 \pm 50.5,269.5 \pm 52.1 / \mathrm{s} \mathrm{mol}\right.$, respectively). Radiosynthesis of $12-\left[{ }^{18} \mathrm{~F}\right]$ fluorododecanoic aminohexanoicanilide $\left(12-\left[{ }^{18} \mathrm{~F}\right] \mathrm{DDAHA}\right)$ was achieved by nucleophilic radiofluorination of 12-iododecanoic-AHA precursor.

Results: A significantly higher accumulation of $12-\left[{ }^{18} \mathrm{~F}\right] \mathrm{DDAHA}$ was observed in MCF-7 and MDA-MB-435 cells in vitro as compared to U87, MiaPaCa, and MCF10A, which was consistent with levels of SIRT2 expression. Initial in vivo studies using $12-\left[{ }^{18} \mathrm{~F}\right] \mathrm{DDAHA}$ conducted in a $9 \mathrm{~L}$ glioma-bearing rats were discouraging, due to rapid defluorination of this radiotracer upon intravenous administration, as evidenced by significant accumulation of $\mathrm{F}-18$ radioactivity in the skull and other bones, which confounded the interpretation of images of radiotracer accumulation within the tumor and other regions of the brain.

Conclusions: The next generation of SIRT2-specific radiotracers resistant to systemic defluorination should be developed using alternative sites of radiofluorination on the aliphatic chain of DDAHA. A SIRT2-selective radiotracer may provide information about SIRT2 expression and activity in tumors and normal organs and tissues, which may help to better understand the roles of SIRT2 in different diseases.
\end{abstract}

Key words: SIRT2, Epigenetic Regulation, F-18, Radiotracer, Positron Emission Tomography, Brain

Electronic supplementary material The online version of this article (https:// doi.org/10.1007/s11307-017-1149-8) contains supplementary material, which is available to authorized users.

Correspondence to: Juri Gelovani; e-mail: juri.gelovani@wayne.edu

\section{Introduction}

Over the past two decades, epigenetic regulation has become a rapidly growing, highly innovative and influential field of biology and medicine. One key epigenetic regulatory 
mechanism involves acetylation and deacetylation of lysine residues of histone core proteins and other critical proteins involved in regulation of gene expression and cell function. Protein acetylation and deacetylation is mediated by histone acetylase transferases (HATs) and histone deacetylases (HDACs), respectively. There are currently 18 HDAC proteins identified in the human genome, which are subdivided into 4 main classes [1]. Classes I, II (a and b), and IV are $\mathrm{Zn}^{2+}$ dependent enzymes [2] and include HDACs 1-11, while class III is comprised of seven isoforms of $\mathrm{NAD}^{+}$-dependent HDAC enzymes. HDAC class III enzymes are termed the "silent information regulators" or "sirtuins" (SIRTs), originating from the yeast SIR2 homologue, and consist of SIRT1-7 [1]. The SIRT isoforms vary in their cellular localization, with SIRTs 1 , 6 , and 7 being primarily located in the nucleus, SIRTs 3 , 4, and 5 located in mitochondria. SIRT2 is primarily localized in the cytoplasm, but can translocate into the nucleus [3] and deacetylase of cytoplasmic alpha-tubulin and nuclear H4K16 proteins, thereby acting as a mitotic exit and cell cycle regulator [3-8].

Several studies have revealed important roles of SIRT2 in the pathogenesis of various diseases. SIRT2 can play a neuroprotective role in neuorodegenerative diseases, such as Parkinson's, Alzheimer's [9], and Huntington's [10]. SIRT2 has been identified as having both cancer-promoting functions (i.e., through stabilization of Myc oncoproteins in breast cancer [11, 12]), as well as cancer-suppressing functions (i.e., through tubulin regulation [4]). Therefore, SIRT2 has been identified as a potential drug target for treatment of various neurodegenerative and inflammatory diseases and cancer. However, it is important to assess its activity during pharmacomodulation (i.e., activation or inhibition) due to pleiotropic roles of SIRT2 under normal and pathologic conditions. The development of non-invasive molecular imaging approaches for monitoring SIRT2 expression and activity in vivo using PET with SIRT2-selective substrate radiotracer may aid in the development of therapies targeting SIRT2.

Understanding structure-activity relationships (SAR) of SIRT2 and its endogenous substrates is important for the development of SIRT2-specific substrate-type radiotracers. The best-studied endogenous substrates of SIRT2 are acetyl$\alpha$-tubulin and acetyl-H4K16. SIRT2, similar to other SIRT isoforms namely 4,5 , and 6 , is able to cleave larger moieties outside of the acetyl. In particular, it has been demonstrated that SIRT2 can cleave a myristoyl group from the myristoyl(K19 and K20)TNF $\alpha$ [13].

To explore the efficiency with which SIRT2 cleaves larger leaving groups, a focused library of potential substrates was synthesized with tert-butyloxycarbonyl-lysine-aminomethylcoumarin (Boc-Lys-AMC) backbone derivatized on the free amino terminus of lysine with a fluoroalkyl chain varying between 3 and 16 carbons in length. Additionally, the influence of fluorine substitution on the $\omega$-carbon in the alkyl chain on substrate affinity and specificity for SIRT2 was evaluated as well. The library of compounds was studied using a fluorogenic enzyme assay with recombinant SIRT2 to determine trends in catalytic efficiency. Furthermore, the SAR of these compounds with SIRT2 was investigated using in silico modeling to better understand the observed trends (Fig. S1 Electronic Supplementary Material (ESM)). Based on these studies, the lead compound, the Boc-Lys(12-fluorododecanoyl)-AMC, was identified and its analogue with an aminohexanoicanilide (AHA) backbone was synthesized (12-fluorododecanoicAHA, 12-FDDAHA). The AHA backbone has been used previously to generate effective HDAC class IIa-specific radiotracers: $\left[{ }^{18} \mathrm{~F}\right] \mathrm{FAHA}$ and $\left[{ }^{18} \mathrm{~F}\right]$ TFAHA $[14,15]$. Additionally, the radiolabeled analogue of the lead compound, 12- $\left[{ }^{18} \mathrm{~F}\right] \mathrm{DDAHA}$, was synthesized from 12-iodododecanoic and 12-bromododecanoic precursors. Finally, the uptake of $12-\left[{ }^{18} \mathrm{~F}\right]$ DDAHA was studied in vitro to determine the magnitude of radiotracer accumulation in cells with high versus low SIRT2 expression. However, initial in vivo studies using $12-\left[{ }^{18} \mathrm{~F}\right]$ DDAHA conducted in a 9L gliomabearing rats were discouraging, due to rapid defluorination of this radiotracer upon intravenous administration, as evidenced by significant accumulation of F-18 radioactivity in the skull and other bones, which confounded the interpretation of images of radiotracer accumulation within the tumor and other regions of the brain. Due to these observations, full characterization of this radiotracer in vivo (i.e., blocking studies) has not been performed. The next generation of SIRT2-specific radiotracers with reduced systemic defluorination should be developed using alternative sites of radiofluorination on the aliphatic chain of DDAHA (i.e., the 3-position).

\section{Materials and Methods}

\section{Synthesis of $\omega$-Fluorinated Acyl Chains}

Details of chemical synthesis and analytical procedures can be found in Electronic Supplementary Materials (ESM) online.

\section{SIRT Enzyme Assay}

Recombinant SIRT1-7 enzymes and BPS1 reference substrate for SIRT2, an oligopeptide corresponding to 379-382 of p53 (Arg-His-Lys-Lys(Ac)-AMC) [16, 17] were purchased from BPS Bioscience (San Diego, CA) and tested using fluorogenic assay. Further details can be found in ESM online.

\section{Computational Docking Studies}

Schrodinger Suite (New York, NY) with the Glide [18] docking program was used for simulating ligand-protein interactions in silico. Further details can be found in ESM online. 


\section{Cell Cultures and In Vitro Radiotracer Uptake Studies}

The U87MG, MCF-7, MDA-MB-435, and MiaPaca cell lines were used to demonstrate differential levels of 12$\left[{ }^{18} \mathrm{~F}\right] \mathrm{DDAHA}$ cellular accumulation. Further details can be found in ESM online.

\section{Intracerebral Glioma Model in Rats}

9L tumor cells were implanted into Sprague-Dawley rats and grown in vivo for development of a glioma-bearing rat model. Further details can be found in ESM online.

\section{PET/CT/MR Imaging}

PET imaging with 12-[ $\left[{ }^{18} \mathrm{~F}\right]$ DDAHA was conducted in a similar manner as previously published [15]. Details can be found in the ESM online.

\section{Histology and Immunohistochemistry}

After the completion of in vivo imaging studies, animals were euthanized under anesthesia and the brain was extracted and prepared for immunohistochemical staining to visualize SIRT2 expression. Details can be found in ESM online.

\section{Results}

The library of compounds with terminally fluorinated acyl chains was synthesized through traditional coupling methods of the fluorinated carboxylic acid chain with the lysine amino derivative via in-situ formation of an acetyl chloride in moderate yields of 20-55\%. Synthesis of the fluorinated carboxylic acid chains employed a novel approach: diethylaminosulfur trifluoride was used for non-selective fluorination of the $n$-hydroxycarboxylic acid forming the acetyl fluoride and fluorination of the terminal carbon. Selective hydrolysis of the acetyl fluoride was achieved using flash chromatography over silica gel to form a mono$\omega$-fluorinated chain due to the abrupt change in $\mathrm{pH}$. The purity of final compounds in the library was $>95 \%$, as determined by ${ }^{1} \mathrm{H}-\mathrm{NMR},{ }^{13} \mathrm{C}-\mathrm{NMR},{ }^{19} \mathrm{~F}-\mathrm{NMR}$, and HRMS (Fig. 1a).

Compound 7 was synthesized from Cbz-Lys-OH (Bachem) and Boc-Lys-AMC (Bachem), which was derivatized to Boc-Lys(myr)-AMC. Following myristoylation, the Boc protecting group was removed and the resulting free lysine amino terminus was coupled with the Cbz-Lys-OH group. The four-step synthesis yielded 7 in a yield of $12 \%$ with $>95 \%$ purity as determined by ${ }^{1} \mathrm{H}-$ NMR, ${ }^{13}$ C-NMR, and HRMS (Fig. 1b).

\section{Radiosynthesis}

12-Fluorododecanoyl-AHA (12-FDDAHA) and 12-[ $\left.{ }^{18} \mathrm{~F}\right]-$ dodecanoylAHA (12-[ $\left.\left[{ }^{18} \mathrm{~F}\right] \mathrm{DDAHA}\right)$ were synthesized as non-radiolabeled and radiolabeled analog of 4 (Fig. 1c-e, $\mathrm{S} 1 \mathrm{ESM})$. The initial radiosynthesis of $12-\left[{ }^{18} \mathrm{~F}\right] \mathrm{DDAHA}(\mathbf{1 1})$ was performed using a brominated precursor, 12Br-DDAHA (9); however, due to low radiochemical yield, the iodinated precursor, 12I-DDAHA (10), was used for optimization of the radiolabeling procedure and improvement of radiosynthetic yield. The iodinated precursor (10) was synthesized from the brominated precursor in a high yield of $73 \%$ using literature procedure [19]. The radiochemical yield achieved with precursor $\mathbf{1 0}$ was 5-fold higher than that with precursor 9. The highest radiofluorination yields were achieved using precursor $\mathbf{1 0}$ in acetonitrile at $85-90{ }^{\circ} \mathrm{C}$. $12-$ $\left[{ }^{18} \mathrm{~F}\right] \mathrm{DDAHA}$ was obtained with purity of $>95 \%$ (Fig. 1d, and S3 ESM) and a decay-corrected radiochemical yield of $12 \%$ (total preparation time $66 \mathrm{~min}$ ) with specific activity of $259 \mathrm{GBq} / \mathrm{mmol}$.

\section{Biochemical Characterization}

Results from in vitro characterization of this library of compounds using fluorogenic assay demonstrated a positive correlation between the substrate efficiency for SIRT2 and the increasing length of the leaving alkyl chain. Compound 5 containing the myristoyl group was the most efficient substrate of SIRT2 with $K_{\text {cat }} / K_{\mathrm{m}}=715.6 \pm 72.8 / \mathrm{s}$ mol followed by compound 4 (fluorododecanoyl) and compound $\mathbf{3}$ (fluorodecanoyl) with $K_{\text {cat }} / K_{\mathrm{m}}$ of $615.4 \pm 50.5 / \mathrm{s} \mathrm{mol} \mathrm{and} 269.5$ $\pm 52.1 / \mathrm{s}$ mol, respectively. In contrast, compound 6 containing the fluoropalmitoyl leaving group exhibited a significant decrease in substrate efficiency for SIRT2 (30.4 $\pm 7.3 / \mathrm{s} \mathrm{mol})$, as compared to compounds containing shorter fluoroalkyl chains. The reference substrate, p53(379-382)K382ac-AMC (BPS1) exhibited $K_{\text {cat }} / K_{\mathrm{m}}=275 \pm 42 / \mathrm{s} \mathrm{mol} \mathrm{(Fig.} \mathrm{2,} \mathrm{Table} \mathrm{1).}$

To assess the influence of a "cap" modification on SIRT2 selectivity, compounds $\mathbf{4}, \mathbf{5}$, and $\mathbf{7}$ were evaluated against a panel of recombinant sirtuins (SIRT1-7). Compound 7 was cleaved efficiently by SIRT2, SIRT3, and, to a lesser extent, by SIRT6. In contrast, $\mathbf{4}$ and $\mathbf{5}$ were cleaved efficiently only by SIRT2 (Fig. S2 ESM). To validate the substrate efficiency of 4 with SIRT2, a competitive inhibition assay with AGK2 (SIRT2-specific inhibitor) was performed, which yielded an $\mathrm{IC}_{50}$ of $28.21 \mu \mathrm{mol}$ of AGK2 (Fig. S2 ESM).

\section{In Silico Modeling}

In silico modeling studies demonstrated that positioning of compounds $\mathbf{4}$ and $\mathbf{5}$ in the SIRT2 active site resulted in a smaller distance between the carbonyl carbon and the nicotinamide-ribose ester linkage (Fig. S4a and S4b ESM). This may contribute to enhanced substrate efficiency of these compounds. 

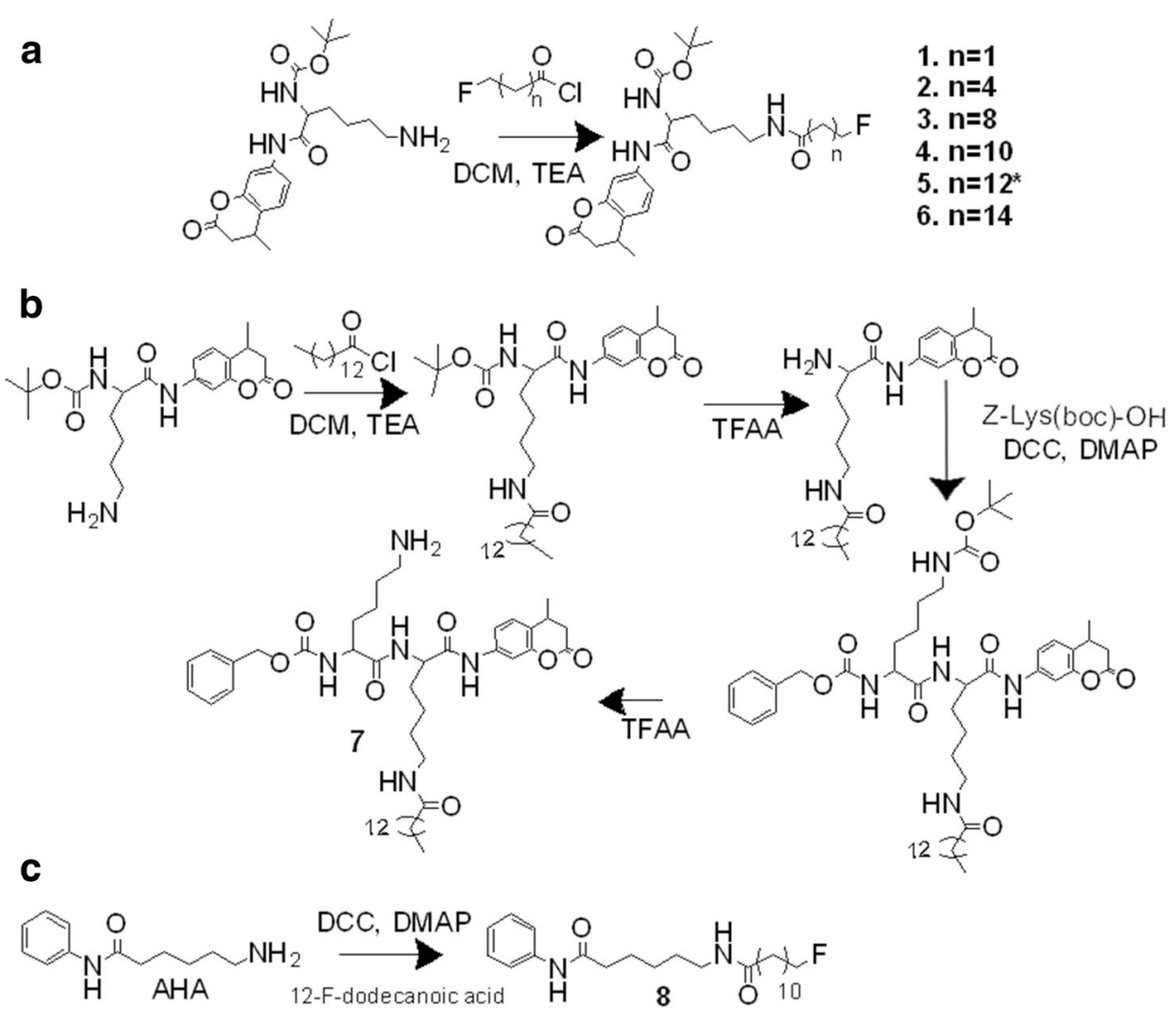

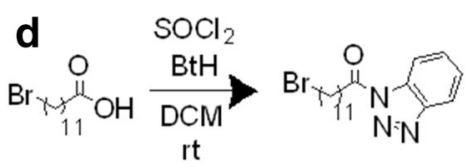

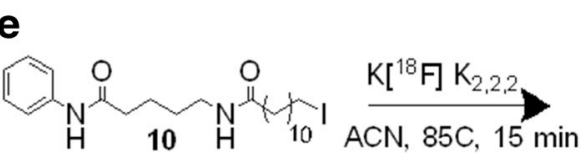

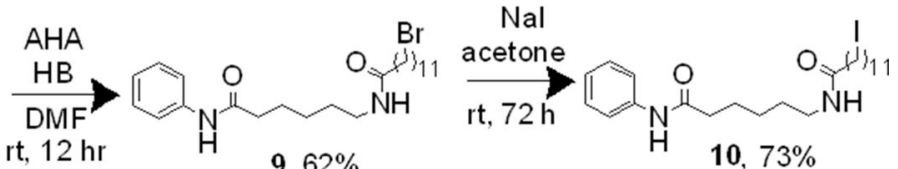

9. $62 \%$<smiles>O=C(CCCNC(=O)C(=O)Nc1ccccc1)NC(F)(F)F</smiles>

Fig. 1. a The focused library of compounds synthesized with fluroalkyl chain-derivatized lysines. The backbone used tertbutyloxycarbonyl-L-lysine-7-amino-3-methylcoumarin (Boc-Lys-AMC) was coupled to fluoroalkyl chains, varying in length from 3 carbons to 16 carbons using acetyl chloride coupling. b To experimentally determine the effect of the cap on the selectivity of a substrate for SIRT2, we developed a di-lysine substrate, with a more peptidic backbone, Cbz-lys-lys(myr)-AMC. This allows for head-to-head comparison between the Boc-lys(myr)-AMC. c The synthesis of both the cold (non-radiolabeled) analogue (9) and $\mathbf{d}$ precursors for $12-\left[{ }^{18} \mathrm{~F}\right] \mathrm{DDAHA}, 12-\operatorname{BrDDAHA}(\mathbf{9})$, and 12-IDDAHA (10). e The 12- $\left[{ }^{18} \mathrm{~F}\right] \mathrm{DDAHA}$ (11) radiotracer was synthesized from the iodinated precursor and the in dry acetonitrile in $85^{\circ} \mathrm{C}$ for $15 \mathrm{~min}$.

\section{In Vitro Radiotracer Accumulation Studies}

MDA-MB-435 and MCF-7 with high levels of SIRT2 expression [20] demonstrated significantly higher levels of 12- $\left[{ }^{18}\right.$ F]DDAHA accumulation (Fig. 3), as compared to $\mathrm{U} 87 \mathrm{MG}>\mathrm{MiaPaCa}>\mathrm{MCF} 10 \mathrm{~A}$ cells, which are known to express lower levels of SIRT2 [20] (very low levels of SIRT2 in U87 reported in Maxwell, et al. 2011 [21]). The extracellular-to-intracellular radioactivity concentration equilibrium was achieved in about $1 \mathrm{~min}$ of incubation with 12 $\left[{ }^{18} \mathrm{~F}\right] \mathrm{DDAHA}$, as the result of high lipophilicity of this radiotracer $\operatorname{cog} \underline{P}=5.32$ which facilitates passive diffusion of intact (parent) compound across the cell membranes in and out of the cells (data not shown). The accumulation and retention of $12-\left[{ }^{18} \mathrm{~F}\right]$ DDAHA-derived radioactivity is due to much higher negative polarity of ADPR-O$\left[{ }^{18} \mathrm{~F}\right]$ dodecanoate, which is the theoretical product of enzymatic reaction of SIRT2 and 12-[ ${ }^{18}$ F]DDAHA.

\section{PET/CT/MR Imaging}

Dynamic PET/CT/MR imaging of a 9L glioma tumorbearing rat with $\left[{ }^{18} \mathrm{~F}\right]-12-D D F A H A$ demonstrated increased 

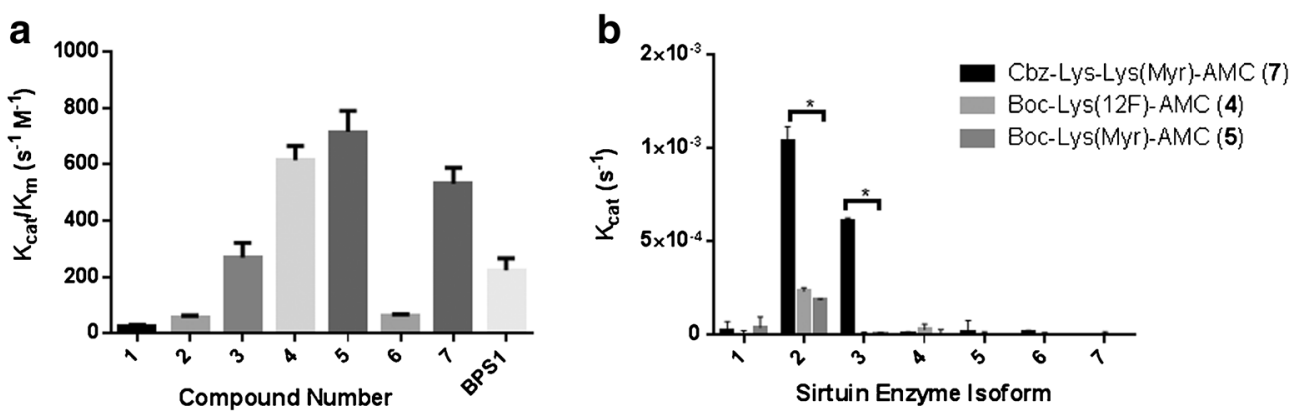

Fig. 2. Biochemical assay data from Fluor de Lys assay®. a The catalytic efficiency $\left(K_{\text {cat }} / K_{m}\right)$ for SIRT2 against different length fluoroalkyl chain leaving groups to assess the structure activity relationship between these compounds and SIRT2. The error bars represent the standard deviation of $N=6$ experiments. These values were taken from 15-min incubation to capture linear phase kinetics. b Compound $\mathbf{7}$ is not selective for SIRT2 whereas compounds $\mathbf{4}$ and $\mathbf{5}$ are selective for SIRT2. These results were taken from 40-min incubation of substrate with enzyme. The error bars represent the standard deviations of the means with significance of $P<0.05$ denoted by *, as determined by two-way ANOVA. c The table details the effect on $K_{\mathrm{m}}$ (amount of substrate necessary for half of $V_{\max }$ ), $V_{\max }$ (the maximal turnover velocity for a given substrate in SIRT2), and $K_{\text {cat }}$ (the catalytic efficiency for a given substrate with SIRT2). This data is determined in linear phase kinetics of SIRT2 with 15-min of incubation; therefore, this data is slightly different than the values in Fig. $2 \mathrm{~b}$.

tracer derived-radioactivity accumulation and retention within the tumor versus normal brain structures between 15 and 20 min post i.v. administration (Fig. 4). However, interpretation of PET/CT/MR images of $\left[{ }^{18} \mathrm{~F}\right]-12-D D F A H A-$ derived radioactivity accumulation in $9 \mathrm{~L}$ gliomas and in the brain cortex was challenging due to high levels of radioactivity accumulation in the adjacent scull bones, which created "spillover" artifacts (Fig. 4b, c). High levels of radioactivity in the bone were caused by rapid defluorination of $\left[{ }^{18} \mathrm{~F}\right]-12$-DDFAHA after i.v. administration. To reduce the appearance of bone radioactivity-causing artifacts in brain PET images, we used the NIH + white (max) lookup table for color coding the range of radioactivity concentrations in the images (Fig. 4d).

\section{Immunohistochemical Validation of SIRT2 Expression in 9L Glioma and Normal Brain Structures}

Fluorescence microscopy of sections of rat brains revealed heterogeneously distributed patchy appearing circularshaped regions of SIRT2 expression in 9L gliomas (Fig. 5a, b), as well as in the peripheral infiltrating zones.
Immunihistochemical staining for HIF-1a expression in adjacent tissue sections confirmed that the areas of reduced SIRT2 expression in tumors co-localized with areas of increased HIF-1a expression (Fig. 5c, d). In the cerebral cortex, SIRT2 expression was expressed mainly in the neurons, with the highest concentrations in perinuclear regions of pyramidal neurons and lower levels in the axons (Fig. 5e, f). In the hippocampus, the level of SIRT2 expression was highest in CA2 and CA3 neurons and had a similar sub-cellular perinuclear localization pattern, as in cortical neurons (Fig. 5g, h). In the cerebellum, SIRT2 was strongly overexpressed in Purkinje cells, with perinuclear localization stronger than in cortical and hippocampal neurons; SIRT2 was also observed in neurons in the molecular layer at somewhat lower levels of expression than in Purkinje cells, but was almost entirely absent in the granular layer neurons (Fig. 5i, j).

\section{Discussion}

To develop the first-generation substrate-type radiotracer selective to SIRT2, it was necessary to understand the structure-activity relationships of SIRT2 with various natural

Table 1. Kinetic parameters for SIRT2 and various chain length compounds

\begin{tabular}{|c|c|c|c|c|c|c|c|c|}
\hline Compound & $K_{\mathrm{m}}(\mu \mathrm{M})$ & SD & $V_{\max }(\mu \mathrm{M} / \mathrm{s})$ & SD & $K_{\text {cat }}(/ \mathrm{sec})$ & SD & $K_{\mathrm{cat}} / K_{\mathrm{m}}(/ \mathrm{s} \mathrm{mol})$ & SD \\
\hline Boc-Lys(3-F-propanoyl)-AMC (1) & 55.53 & 11.4 & 0.15 & 0.016 & $1.41 \times 10^{-3}$ & $5.0 \times 10^{-4}$ & 26.3 & 5.1 \\
\hline Boc-Lys(6-F-hexanoyl)-AMC (2) & 44.89 & 4.56 & 0.28 & 0.013 & $2.58 \times 10^{-3}$ & $4.1 \times 10^{-4}$ & 57.4 & 5.2 \\
\hline Boc-Lys (10-F-decanoyl)-AMC (3) & 18.03 & 4.07 & 0.52 & 0.041 & $4.86 \times 10^{-3}$ & $1.3 \times 10^{-4}$ & 269.5 & 52 \\
\hline Boc-Lys(12-F-dodecanoyl)-AMC (4) & 8.07 & 0.79 & 0.54 & 0.014 & $4.97 \times 10^{-3}$ & $4.2 \times 10^{-4}$ & 615.4 & 50 \\
\hline Boc-Lys (myristoyl)-AMC (5) & 2.36 & 0.22 & 0.26 & 0.010 & $2.38 \times 10^{-3}$ & $3.0 \times 10^{-4}$ & 715.5 & 73 \\
\hline Boc-Lys(palmitoyl)-AMC (6) & 51.15 & 6.91 & 0.33 & 0.022 & $3.09 \times 10^{-3}$ & $6.8 \times 10^{-4}$ & 60.4 & 7.3 \\
\hline Cbz-Lys-Lys(myr)-AMC (7) & 10.36 & 1.30 & 0.50 & 0.022 & $5.51 \times 10^{-3}$ & $6.9 \times 10^{-4}$ & 532.3 & 56 \\
\hline BPS1 & 31.73 & 5.57 & 0.95 & 0.022 & $8.78 \times 10^{-3}$ & $7.0 \times 10^{-4}$ & 275.0 & 43 \\
\hline
\end{tabular}




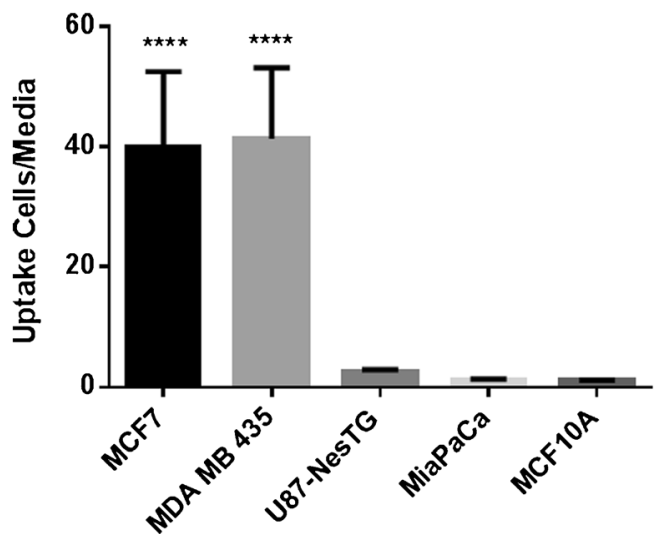

Fig. 3. The radiotracer 12-[ $\left[{ }^{18} \mathrm{~F}\right] \mathrm{DDAHA}$ (11) was used in cellular uptake studies for tumor cell lines. The error bars represent the standard deviation for $N=4$ for each cell line. Where ${ }^{* * \star *}$ represents $P<0.05$ as determined by one-way ANOVA and Tukey's multiple comparisons test, this value is significantly larger than the other three values.

and synthetic substrates. Previously, it has been reported that in addition to an acetyl group, SIRT2 can also cleave long acyl chains from lysine residues, including a myristoyl moiety [22]. Other HDAC class III enzymes, such as SIRT3 and SIRT6, have also been reported to cleave myristoylated lysines of the H3K9 peptide [23, 24] and K19-20TNF- $\alpha$ [22]. However, there are no data on the effects of fluorine substitution on the $\omega$-carbon in alkyl chains on different lengths on SIRT2 catalytic efficiency.

Our current studies demonstrate that catalytic efficiency of SIRT2 gradually increases with the elongation of fluoroalkyl chain from 3 to 12 carbons. The catalytic efficiency of SIRT2 for fluorododecanoyl chain $\left(K_{\text {cat }} / K_{\mathrm{m}}=\right.$ $615.4 \pm 50.5 / \mathrm{s} \mathrm{mol}$ ) is comparable (difference is not statistically significant) with that of the non-fluorinated myristoyl chain (reference compound, BPS1), which is cleaved most efficiently $\left(K_{\text {cat }} / K_{\mathrm{m}}=716.5 \pm 72.8 / \mathrm{s} \mathrm{mol}\right)$. In contrast, the catalytic efficiency of SIRT2 was significantly lower for the fluoropalmitoyl group $\left(K_{\text {cat }} / K_{\mathrm{m}}=60.4 \pm 7.3 / \mathrm{s} \mathrm{mol}\right)$. These observations agree with previous reports, demonstrating a similar trend in SIRT2 catalytic efficiency for the H3K9 peptide backbone derivatized with hexanoyl, octanoyl, decanoyl, dodecanoyl, and myristoyl groups, but significantly less efficiently the palmitoyl and lipoyl-derivatized H3K9 peptide [23, 24]. Similarly, using TNF- $\alpha$ peptide backbone with varying chain lengths to a fluorophore or quencher moiety the longer chain length (11 carbons) resulted in much improved catalytic efficiency over the shorter (6 or 8 carbons) or very long (13 carbons) chain lengths [25]. However, despite the similarities in trends, the absolute $K_{\text {cat }} / K_{\mathrm{m}}$ values obtained in the current study for different alkyl chains with Boc-Lys-AMC backbone are significantly lower than those reported previously using a H3K9 or TNF- $\alpha$ peptide [23-25], which can be explained, at least in part, by the higher binding efficiency of a peptide "cap" group, as compared to Boc-Lys-AMC. Similarly, reports of a "cap" group containing either p53 or TNF- $\alpha$ peptide residues on one side and an AMC group on the other demonstrate reduced $K_{\text {cat }} / K_{\mathrm{m}}$ as compared to compounds with a full peptide cap $[23,25,26]$. Also, the observed differences may be due to the presence of electronegative $\omega$ terminal fluorine. In the current study, the $K_{\text {cat }} / K_{\mathrm{m}}$ values were determined by measuring the rate of the AMC cleavage product formation [26], whereas in some of the previously reported data, $K_{\text {cat }} / K_{\mathrm{m}}$ values were determined by measuring the rate of $\mathrm{NAD}^{+}$consumption, which may also explain the differences in absolute $K_{\text {cat }} / K_{\mathrm{m}}$ values between studies [23].

To examine the selectivity of compounds for SIRT2 among all SIRT enzymes, three of the most efficient substrates, 4, 5, and $\mathbf{7}$ were screened against a panel of recombinant SIRT enzymes (SIRT1-7). The aim was to elucidate the differences in SIRT selectivity that may occur as a result of altering the cap (mono- $v s$ di-lysine cap), the leaving group (12- vs 14-carbon chain), and last the terminal fluorine atom (12F-carbon vs 14-carbon). Our results indicate that compound 7 , with a di-lysine cap, was cleaved more effectively by SIRT3 and SIRT6 than compound $\mathbf{5}$, with a mono-lysine cap. Therefore, $\mathbf{5}$ is more selective to SIRT2 as it shows very little cleavage by other SIRT enzymes. Both 4 (12F-carbon chain) and 5 (14carbon chain) are cleaved effectively by SIRT 2 and not by any other SIRT enzymes, indicating that both leaving groups are selective for SIRT2. These findings are consistent with other reports in the literature [22, 24, 27] and demonstrate that leaving group specificity may not entirely define the selectivity of a substrate to a certain SIRT enzyme. Altering the cap group can have a significant impact on the efficiency with which the SIRT enzyme cleaves a substrate.

Also, we assessed the effectiveness of the lead compound $\mathbf{4}$ as a synthetic substrate for SIRT2 using a potent selective SIRT2 inhibitor AGK2 [28]. A competitive inhibition study [28] was performed using increasing concentrations of AGK2 with 4 at its apparent $K_{\mathrm{m}}$ concentration $(8 \mu \mathrm{M})$ for SIRT2. The observed inhibition of SIRT2 by AGK2 at $\mathrm{IC}_{50}$ of $28.21 \mu \mathrm{M}$ using 4 as substrate was comparable to previously reported $\mathrm{IC}_{50}$ values of $3-$ $5 \mu \mathrm{M}$ obtained with myristoylated or acetylated substrates of SIRT2 [29, 30]. As demonstrated by the comparable catalytic efficiencies of 4 and BPS1 (p53 (379382)K382ac-AMC) this data further confirm that 4 is an efficient substrate of SIRT2.

Based on the results of biochemical and in silico modeling studies (additional discussion can be found in Supplemental Materials), we have chosen the dodecanoyl chain fluorinated on the $\omega$-position for coupling to an AHA backbone (Fig. S2 ESM) to generate the SIRT2specific ${ }^{18} \mathrm{~F}$-labeled radiotracer termed $12-\left[{ }^{18} \mathrm{~F}\right]$ DDAHA. We did not study the 14-fluoromyristoyl-Boc-Lys-AMC and $14-\left[{ }^{18} \mathrm{~F}\right]$ fluoromyristoyl-AHA, because Boc-Lys(12fluorododecanoyl)-AMC exhibits $2 / 3$ efficiency of Boc- 

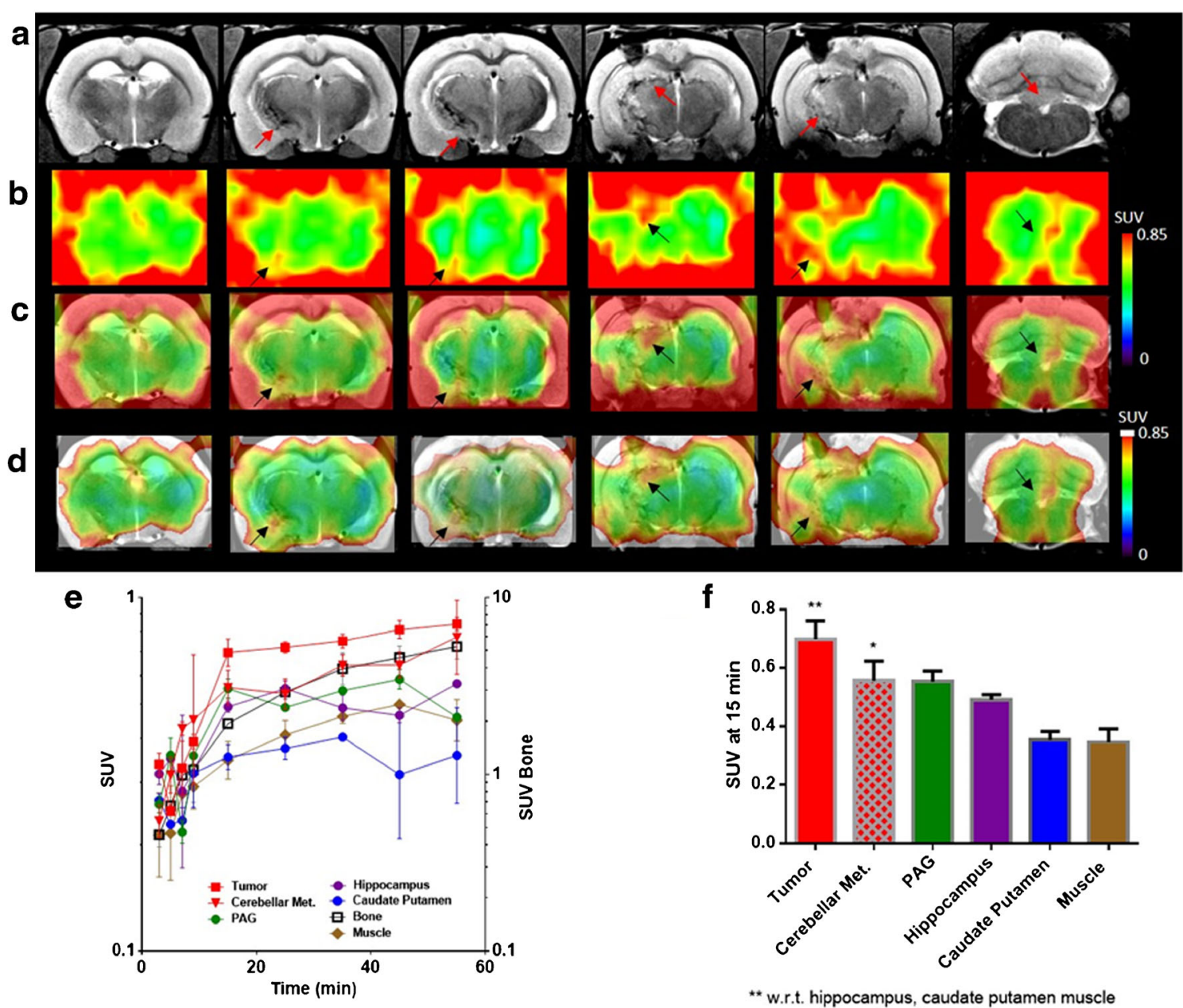

** w.r.t. hippocampus, caudate putamen muscle

* w.r.t. muscle

Fig. 4. a T2-weighted MR image sliced coronally through the $9 \mathrm{~L}$ glioma lesion and hippocampus with red arrows indicating areas of tumor lesion. b Dynamic PET images at 15-min post administration of 12-[ ${ }^{18}$ F]DDAHA NIH lookup table (LUT). c Dynamic PET images post administration of $12-\left[{ }^{18} \mathrm{~F}\right]$ DDAHA overlaid onto T2-weighted MR images using the NIH LUT. d The same images as in c, but with the NIH + white LUT, to better visualize the internal structures due to the high degree of defluorination occurring, which is seen as F-18 accumulation in the bone causing a "halo" effect around the cortical region due to partial volume effect of bone signal into cortex. e Time activity curves for each region of interest within the brain over 60 min of dynamic PET images displayed as log (SUV) vs time. The error bars represent standard deviation of the voxel values within each ROI. $\mathbf{f} A$ visual representation of differential washout between tumor and other regions of the brain at 15-min post i.v. administration of $12-\left[{ }^{18} \mathrm{~F}\right] \mathrm{DDAHA}$, where error bars represent standard deviations and * or ${ }^{*}$ represent $P<0.05$ as found from one-way ANOVA.

Lys(myristoyl)-AMC as selective substrate for SIRT2 and because radiotracers with smaller molecular weight have pharmacokinetic advantages over radiotracers with larger molecular weight. The AHA backbone was chosen to increase the lipophilicity of the radiotracer and permeability across cell membranes, which would not be possible with a larger peptidic backbone. Accordingly, the radiotracer $12-\left[{ }^{18} \mathrm{~F}\right] \mathrm{DDAHA}$ has a cLogP value of 5.32 indicating a strong ability to passively cross the cell membrane in and out of the cell. While the change in "cap" group may alter the selectivity to SIRT2, it has a lesser effect on the catalytic efficiency, as this is determined largely by the leaving group structure rather than the cap group. Nevertheless, the synthesis and evaluation of these compounds are currently underway and will be reported separately. For synthesis of 12$\left[{ }^{18} \mathrm{~F}\right] \mathrm{DDAHA}$, we adapted methods for radiolabeling of $\omega-\left[{ }^{18} \mathrm{~F}\right]$ substituted long-chain fatty acids $[19]$ that are used for PET imaging of cardiac metabolism [31]. Our studies confirmed previous reports that $\omega$-iodinated dodecanoyl precursor provides a 3 -fold higher radiosynthetic yield than the $\omega$-brominated precursor [19]. The radiosynthetic yield for $12-\left[{ }^{18} \mathrm{~F}\right]$ DDAHA could be optimized further by using microwave-assisted heating [19].

In vitro radiotracer uptake studies in cell lines with varying degrees of SIRT2 expression provided initial 

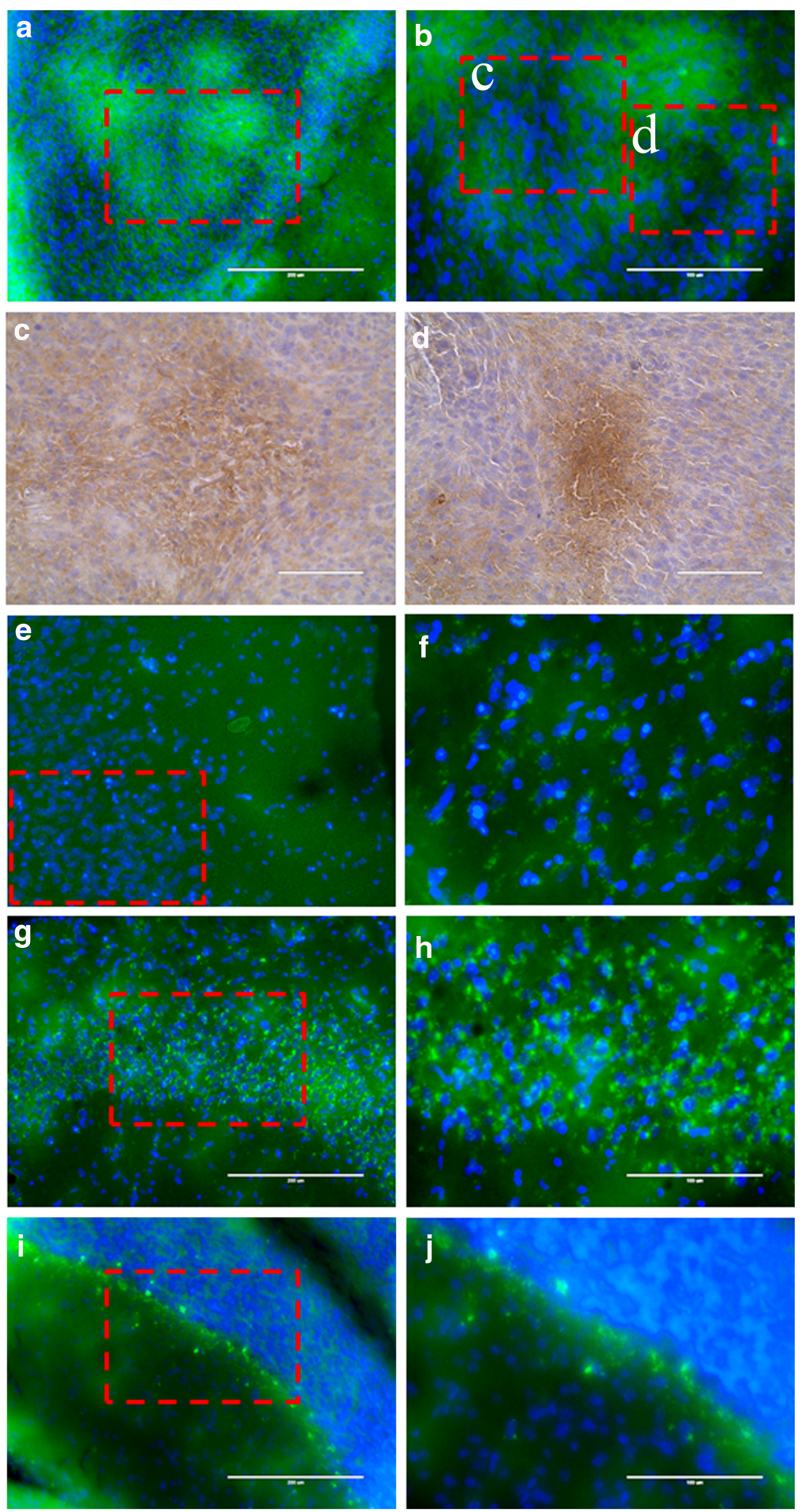

Fig. 5. Fluorescence and light microscopy images of different regions of immunostained tissue sections of the rat brain with 9L gliomas obtained at $\times 200(\mathbf{a}, \mathbf{c}, \mathbf{e}, \mathbf{g}, \mathbf{i})$ and $\times 400(\mathbf{b}, \mathbf{d}, \mathbf{f}, \mathbf{h}, \mathbf{j})$. Immunofluorescent images of SIRT2 expression in (a, b) 9L glioma, (e, f) contralateral brain cortex, (g, h) CA2-3 region of contralateral hippocampus, and (i, j) cerebellum. Immunohistochemically stained adjacent tissue sections of intracerebral 9L gliomas reveal high levels of HIF-1 $\alpha$ expression (c, d) corresponding to regions of decreased SIRT2 expression in panel. 
evidence for the efficacy of $12-\left[{ }^{18} \mathrm{~F}\right] \mathrm{DDAHA}$ as a radiotracer for quantification of SIRT2 expression-activity. The level of 12-[ ${ }^{18}$ F]DDAHA-derived radioactivity accumulation was significantly increased in MDA-MB-435 melanoma and MCF-7 breast adenocarcinoma and to a much lesser degree, in U87MG glioma, as compared to $\mathrm{MiaPaCa}$ pancreatic carcinoma and MCF10A immortalized breast epithelial cells. MCF-7 cells are known to have increased SIRT2 expression [20, 32]. Also, MiaPaCa cells have demonstrated increased levels of SIRT2 expression via Western blot analysis [11]. The non-tumorigenic epithelial cells, MCF10A, exhibit significantly lower levels of SIRT2 expression [20] and served as a lower-expressing control for this study. These results indicate levels of $12-\left[{ }^{18} \mathrm{~F}\right] \mathrm{DDAHA}$-derived radioactivity accumulation correspond to levels of SIRT2 expression as reported elsewhere for MCF-7, MDA-MB-435, U87MG, MiaPaCa, and MCF10A cell lines.

Despite the high lipophilicity of the fluoroalkyl leaving group, the mechanism of substrate cleavage by SIRT enzymes allows for a radioactive leaving group to be entrapped in the cell, at least transiently. Specifically, SIRT2 enzymatic cleavage mechanism results in formation of an ester linkage between the alkyl leaving group and $O$-ADPR (Fig. S1 ESM), resulting in ${ }^{18} \mathrm{~F}$-fluoroalkyl-O-ADPR product which cannot cross a cell membrane because it is quite large and very polar (containing 2 negatively charged phosphate groups) (Fig. S1 ESM). Therefore, SIRT2mediated cleavage of ${ }^{18} \mathrm{~F}$-fluoroalkyl group from 12$\left[{ }^{18} \mathrm{~F}\right]$ DDAHA with formation of ${ }^{18} \mathrm{~F}$-fluoroalkyl-O-ADPR complex represents the rate-limiting step in the transient cellular entrapment of $12-\left[{ }^{18} \mathrm{~F}\right] \mathrm{DDAHA}$-derived radioactivity. The eventual efflux of $12-\left[{ }^{18} \mathrm{~F}\right] \mathrm{DDAHA}$-derived radioactivity from intracerebral $9 \mathrm{~L}$ glioma also suggests that ${ }^{18} \mathrm{~F}-$ fluoroalkyl-O-ADPR is degradable and that ${ }^{18} \mathrm{~F}$-fluoroalkyl moiety eventually diffuses out of the cells. The removal of the acetyl or longer acyl chain moieties from $O$-ADPR group has been hypothesized by others to occur by way of ARH3 cleavage or possible methanolysis to form $1^{\prime} O$-methyl' ADPR [33, 34]. Additionally, a nucleophilic attack from lysine side chains of other proteins in close proximity may also de-acetylate the $O$-AADPR. Further studies are needed to fully characterize the half-life of $O-12-\left[{ }^{18} \mathrm{~F}\right] \mathrm{DD}-\mathrm{ADPR}$ and residence time for the $12-\left[{ }^{18} \mathrm{~F}\right]$ dodecanoyl moiety within the cell. Although this entrapment may be transient, it provides a sufficient retention time within the cells with high SIRT2 expression-activity for PET imaging.

Furthermore, our initial in vivo PET imaging studies using $12-\left[{ }^{18} \mathrm{~F}\right]$ DDAHA conducted in a $9 \mathrm{~L}$ glioma-bearing rats were discouraging, due to rapid systemic defluorination of this radiotracer upon intravenous administration. This was evidenced by significant accumulation of F-18 radioactivity in the skull and other bones, which confounded the interpretation of images of radiotracer accumulation within the tumor and other regions of the brain. Therefore, additional PET/CT MR images in panel Fig. 4d are shown in NIH + white (max) look-up table, and the resulting PET/
MRI fusion images revealed 12-[ $\left.{ }^{18} \mathrm{~F}\right] \mathrm{DDAHA}$-derived radioactivity accumulation in 9L gliomas and normal brain regions, such as the hippocampus. This proof of concept imaging study demonstrated adequate BBB permeability, as evidenced by the rapid equilibration of $12-\left[{ }^{18} \mathrm{~F}\right]$ DDAHA radioactivity between blood and normal brain tissue (Fig. 4). Progressive accumulation of $12-\left[{ }^{18} \mathrm{~F}\right]$ DDAHA-derived radioactivity in normal brain structures with high levels of SIRT2 expression and especially in 9L tumor lesions (Fig. 4) during the first 15-min post intravenous administration supports the proposed mechanism of SIRT2-mediated accumulation and transient retention in tissues. Static images of 12-[ $\left.{ }^{18} \mathrm{~F}\right]$ DDAHA accumulation/retention (SUV) obtained between 15 and $20 \mathrm{~min}$ post i.v. injection with highest tumor-to-normal brain and tumor-to-muscle SUV ratios may provide more specific images of SIRT2 expression-activity in target tissues, if defluorination would be minimized in the next generation of radiotracers. Because of the observed excessive defluorination of $12-\left[{ }^{18} \mathrm{~F}\right]$ DDAHA upon intravenous administration, a more complete characterization of this radiotracer in vivo (i.e., blocking studies) has not been performed.

To validate the results of PET/CT/MR imaging with 12$\left[{ }^{18} \mathrm{~F}\right] \mathrm{DDAHA}$ and to assess the cell specificity and heterogeneity of SIRT2 expression-activity in 9L gliomas and in different brain structures, we used immunofluorescent microscopy (IFM) in 9L glioma-bearing rat brain tissue sections. Fluorescence microscopy of tissue sections of intracerebral 9L gliomas growing in the rat brains stained with anti-SIRT2 fluorescent antibodies revealed heterogeneously distributed patchy-appearing circular regions of SIRT2 expression in core regions in peripheral infiltrating zones of 9L gliomas. Such pattern of expression suggested that SIRT2 is upregulated in well-perfused regions of 9L gliomas, whereas the expression of SIRT2 in hypoxicappearing regions is significantly reduced, which was confirmed immunohistochemically by presence of HIF- $1 \alpha$ expression in the same regions in adjacent tissue sections. These results are consistent with previous reports of regulatory loops of HIF-1a-SIRT2, involving HIF-1 $\alpha$ mediated transcriptional repression of SIRT2 expression [35], as well as SIRT2-mediated deacetylation of Lys709, which increases prolyl-hydroxylase binding and ubiquitinmediated degradation of HIF-1 $\alpha$ [36].

In the normal brain structures, the highest level of SIRT2 expression was observed in hippocampal $\mathrm{CA} 2$ and CA3 neurons, followed by pyramidal neurons in cerebral cortex, and cerebellar Purkinje cells. The subcellular localization of SIRT2 was predominantly perinuclear with lower levels of diffuse distribution in the rest of the neuronal cytoplasm and axons. These observations of neuronal-specific subcellular localization of SIRT2 in the brain are in agreement with previous reports [21] which demonstrated that SIRT2 expression in the murine brain is higher in hippocampal CA2-3 neurons, cortical neurons, and in cerebellar Purkinje cells and molecular layer neurons, but not in the granular 
layer neurons or other cells of oligedendroglial lineage. Thus, in situ microscopy results can serve as the basis for interpretation of in vivo PET/CT/MRI images and confirm that the increased levels of radiotracer accumulation in intracerebral 9L gliomas and in certain normal brain structures are mediated by increased SIRT2 expressionactivity levels, as compared to other brain structures expressing lower levels of SIRT2.

Also, this study provides grounds for development of the next generation of SIRT2-specific radiotracers. Several previous studies have reported defluorination of $\omega$ $\left[{ }^{18} \mathrm{~F}\right]$ substituted fatty acids in rodents following intravenous injection [31] but significantly lower levels of defluorination occurred in higher order mammals (i.e. pigs and dogs) and in humans, due to a decrease in level of expression of defluorinating enzymes [31]. An alternative approach to decrease defluorination in a second-generation tracer would be to radiolabel at the $\omega-3$ position of the acyl chain. Additionally, carbon-11 labeling of the dodecanoyl chain could be accomplished similar to ${ }^{11} \mathrm{C}$-palmitate [37, 38]. Another approach to decrease the magnitude of systemic defluorination could involve pre-treatment with miconazole (CYP450 2E1 inhibitor) which was shown effective for ${ }^{18} \mathrm{~F}$ FCWAY [39], which, however, is not practical for clinical translation because of potential for high patient to patient variability. Further radiotracer development is underway and will be reported elsewhere.

SIRT2 is an emerging target for therapy of various neurologic diseases, including neurodegeneration [10], as well as cardiac [40] and metabolic diseases [40, 41]. Many SIRT2-targeted inhibitors are currently in development and entering the early-stage clinical trials, further driving the need for continuation of this work towards development and clinical translation of second-generation SIRT2-selective radiotracer.

\section{Conclusions}

In summary, guided by the results of biochemical and in silico modeling studies of structure-activity relationships between SIRT2 and a peptide-mimetic backbone containing a lysine derivatized with fluoroalkyl chains of different length, we have developed and tested in vitro and in vivo a novel, first-generation, SIRT2-selective substrate-type radiotracer, 12- $\left[{ }^{18} \mathrm{~F}\right] \mathrm{DDAHA}$. Current proof of principal studies, demonstrated suitability for radiofluorinated DDAHA assessment of SIRT2 expression-activity using in vitro radiotracer uptake assays. Molecular imaging with PET using next-generation SIRT2-specific radiofluorinated DDAHAbased radiotracer, which is resistant to defluorination in vivo, should provide valuable information about the location and magnitude of SIRT2 expression and activity in the brain and in tumors. PET imaging may help to determine the mechanistic, therapeutic, and prognostic roles of SIRT2 in different diseases and enable monitoring of SIRT2 targeted therapies.

\section{Compliance with Ethical Standards}

\section{Conflict of Interest}

The authors declare that they have no conflict of interest.

Open Access This article is distributed under the terms of the Creative Commons Attribution 4.0 International License (http:// creativecommons.org/licenses/by/4.0/), which permits unrestricted use, distribution, and reproduction in any medium, provided you give appropriate credit to the original author(s) and the source, provide a link to the Creative Commons license, and indicate if changes were made.

\section{References}

1. Haberland M, Montgomery RL, Olson EN (2009) The many roles of histone deacetylases in development and physiology: implications for disease and therapy. Nat Rev Genet 10(1):32-42. https://doi.org/ $10.1038 /$ nrg 2485

2. De Ruijter AJVG, van Gennip AH, Caron HN et al (2003) Histone deacetylases (HDACs): characterization of the classical HDAC family. Biochem J 370(3):737-749. https://doi.org/10.1042/ bj20021321

3. North BJ, Verdin E (2007) Interphase nucleo-cytoplasmic shuttling and localization of SIRT2 during mitosis. PLoS One 2(8):e784. https://doi.org/10.1371/journal.pone.0000784

4. Hiratsuka M, Inoue T, Toda T, Kimura N, Shirayoshi Y, Kamitani H, Watanabe T, Ohama E, Tahimic CGT, Kurimasa A, Oshimura M (2003) Proteomics-based identification of differentially expressed genes in human gliomas: down-regulation of SIRT2 gene. Biochem Biophys Res Commun 309(3):558-566. https://doi.org/10.1016/ j.bbrc.2003.08.029

5. Inoue T, Hiratsuka M, Osaki M, Oshimura M (2007) The molecular biology of mammalian SIRT proteins: SIRT2 in cell cycle regulation. Cell Cycle 6(9):1011-1018. https://doi.org/10.4161/cc.6.9.4219

6. Inoue T, Hiratsuka M, Osaki M, Yamada H, Kishimoto I, Yamaguchi S, Nakano S, Katoh M, Ito H, Oshimura M (2007) SIRT2, a tubulin deacetylase, acts to block the entry to chromosome condensation in response to mitotic stress. Oncogene 26(7):945-957. https://doi.org/ 10.1038/sj.onc.1209857

7. Inoue T, Nakayama Y, Yamada H, Li YC, Yamaguchi S, Osaki M, Kurimasa A, Hiratsuka M, Katoh M, Oshimura M (2009) SIRT2 downregulation confers resistance to microtubule inhibitors by prolonging chronic mitotic arrest. Cell Cycle Tex 8(8):1279-1291. https://doi.org/10.4161/cc.8.8.8245

8. North BJ, Verdin E (2007) Mitotic regulation of SIRT2 by cyclindependent kinase 1-dependent phosphorylation. J Biol Chem 282(27):19546-19555. https://doi.org/10.1074/jbc.M702990200

9. Wang J, Yu J-T, Tan M-S, Jiang T, Tan L (2013) Epigenetic mechanisms in Alzheimer's disease: implications for pathogenesis and therapy. Ageing Res Rev 12(4):1024-1041. https://doi.org/10.1016/ j.arr.2013.05.003

10. Donmez G, Outeiro TF (2013) SIRT1 and SIRT2: emerging targets in neurodegeneration. EMBO Mol Med 5(3):344-352. https://doi.org/ 10.1002/emmm.201302451

11. Liu PY, Xu N, Malyukova A, Scarlett CJ, Sun YT, Zhang XD, Ling D, Su SP, Nelson C, Chang DK, Koach J, Tee AE, Haber M, Norris MD, Toon C, Rooman I, Xue C, Cheung BB, Kumar S, Marshall GM, Biankin AV, Liu T (2013) The histone deacetylase SIRT2 stabilizes Myc oncoproteins. Cell Death Differ 20(3):503-514. https://doi.org/ $10.1038 /$ cdd. 2012.147

12. McGlynn LM, Zino S, MacDonald AI, Curle J, Reilly JE, Mohammed ZMA, McMillan DC, Mallon E, Payne AP, Edwards J, Shiels PG (2014) SIRT2: tumour suppressor or tumour promoter in operable breast cancer? Eur J Cancer 50(2):290-301. https://doi.org/10.1016/ j.ejca.2013.10.005

13. Yao Y-L, Yang W-M (2011) Beyond histone and deacetylase: an overview of cytoplasmic histone deacetylases and their nonhistone substrates. J Biomed Biotechnol 2011:146493 
14. Yeh H-H, Tian M, Hinz R, Young D, Shavrin A, Mukhapadhyay U, Flores LG, Balatoni J, Soghomonyan S, Jeong HJ, Pal A, Uthamanthil R, Jackson JN, Nishii R, Mizuma H, Onoe H, Kagawa S, Higashi T, Fukumitsu N, Alauddin M, Tong W, Herholz K, Gelovani JG (2013) Imaging epigenetic regulation by histone deacetylases in the brain using PET/MRI with 18F-FAHA. NeuroImage 64:630-639. https:// doi.org/10.1016/j.neuroimage.2012.09.019

15. Bonomi R, Mukhopadhyay U, Shavrin A, Yeh HH, Majhi A, Dewage SW, Najjar A, Lu X, Cisneros GA, Tong WP, Alauddin MM, Liu RS, Mangner TJ, Turkman N, Gelovani JG (2015) Novel histone deacetylase class IIa selective substrate radiotracers for PET imaging of epigenetic regulation in the brain. PLoS One 10(8):e133512. https://doi.org/10.1371/journal.pone.0133512

16. Marcotte PA, Richardson PR, Guo J, Barrett LW, Xu N, Gunasekera A, Glaser KB (2004) Fluorescence assay of SIRT protein deacetylases using an acetylated peptide substrate and a secondary trypsin reaction. Anal Biochem 332(1):90-99. https://doi.org/10.1016/j.ab.2004.05.039

17. Huhtiniemi T, Salo HS, Suuronen T, Poso A, Salminen A, Leppänen J, Jarho E, Lahtela-Kakkonen M (2011) Structure-based design of pseudopeptidic inhibitors for SIRT1 and SIRT2. J Med Chem 54(19):6456-6468. https://doi.org/10.1021/jm200590k

18. Zhu K, Borrelli KW, Greenwood JR, Day T, Abel R, Farid RS, Harder E (2014) Docking covalent inhibitors: a parameter free approach to pose prediction and scoring. J Chem Inf Model 54(7):1932-1940. https://doi.org/10.1021/ci500118s

19. Belanger AP, Pandey MK, DeGrado TR (2011) Microwave-assisted radiosynthesis of [(18)F]fluorinated fatty acid analogs. Nucl Med Biol 38(3):435-441. https://doi.org/10.1016/j.nucmedbio.2010.10.001

20. Jing $\mathrm{H}, \mathrm{Hu} \mathrm{J}, \mathrm{He} B$, Negrón Abril YL, Stupinski J, Weiser K, Carbonaro M, Chiang YL, Southard T, Giannakakou P, Weiss RS, Lin H (2016) A SIRT2-selective inhibitor promotes c-Myc oncoprotein degradation and exhibits broad anticancer activity. Cancer Cell 29(3):297-310. https://doi.org/10.1016/j.ccell.2016.02.007

21. Maxwell MM, Tomkinson EM, Nobles J, Wizeman JW, Amore AM, Quinti L, Chopra V, Hersch SM, Kazantsev AG (2011) The Sirtuin 2 microtubule deacetylase is an abundant neuronal protein that accumulates in the aging CNS. Hum Mol Genet 20(20):3986-3996. https://doi.org/10.1093/hmg/ddr326

22. He B, Hu J, Zhang X, Lin H (2014) Thiomyristoyl peptides as cellpermeable Sirt6 inhibitors. Org Biomol Chem 12(38):7498-7502. https://doi.org/10.1039/C4OB00860J

23. Feldman JL, Dittenhafer-Reed KE, Kudo N, Thelen JN, Ito A, Yoshida M, Denu JM (2015) Kinetic and structural basis for acylgroup selectivity and $\mathrm{NAD}(+)$ dependence in Sirtuin-catalyzed deacylation. Biochemist 54(19):3037-3050. https://doi.org/10.1021/ acs.biochem. 5 b00150

24. Feldman JL, Baeza J, Denu JM (2013) Activation of the protein deacetylase SIRT6 by long-chain fatty acids and widespread deacylation by mammalian sirtuins. J Biol Chem 288(43):31350 31356. https://doi.org/10.1074/jbc.C113.511261

25. Schuster S, Roessler C, Meleshin M, Zimmermann P, Simic Z, Kambach C, Schiene-Fischer C, Steegborn C, Hottiger MO, Schutkowski M (2016) A continuous sirtuin activity assay without any coupling to enzymatic or chemical reactions. Sci Rep 6(1):22643. https://doi.org/10.1038/srep22643

26. Chiang YL, Lin H (2016) An improved fluorogenic assay for SIRT1, SIRT2, and SIRT3. Org Biomol Chem 14(7):2186-2190. https:// doi.org/10.1039/C5OB02609A

27. Pan PW, Feldman JL, Devries MK, Dong A, Edwards AM, Denu JM (2011) Structure and biochemical functions of SIRT6. J Biol Chem 286(16):14575-14587. https://doi.org/10.1074/jbc.M111.218990
28. Cui H, Kamal Z, Ai T, Xu Y, More SS, Wilson DJ, Chen L (2014) Discovery of potent and selective sirtuin 2 (SIRT2) inhibitors using a fragment-based approach. J Med Chem 57(20):8340-8357. https:// doi.org/10.1021/jm500777s

29. Lain S, Hollick JJ, Campbell J, Staples OD, Higgins M, Aoubala M, McCarthy A, Appleyard V, Murray KE, Baker L, Thompson A, Mathers J, Holland SJ, Stark MJR, Pass G, Woods J, Lane DP, Westwood NJ (2008) Discovery, in vivo activity, and mechanism of action of a small-molecule p53 activator. Cancer Cell 13(5):454-463. https://doi.org/10.1016/j.ccr.2008.03.004

30. Hoffmann G, Breitenbücher F, Schuler M, Ehrenhofer-Murray AE (2014) A novel sirtuin 2 (SIRT2) inhibitor with p53-dependent proapoptotic activity in non-small cell lung cancer. J Biol Chem 289(8):5208-5216. https://doi.org/10.1074/jbc.M113.487736

31. Pandey MK, Belanger AP, Wang S, DeGrado TR (2012) Structure dependence of long-chain [(18)F]fluorothia fatty acids as myocardial fatty acid oxidation probes. J Med Chem 55(23):10674-10684. https:// doi.org/10.1021/jm301345v

32. Shah AA, Ito A, Nakata A, Yoshida M (2016) Identification of a selective SIRT2 inhibitor and its anti-breast cancer activity. Biol Pharmaceut Bull 39(10):1739-1742. https://doi.org/10.1248/bpb.b1600520

33. Fahie K, Hu P, Swatkoski S, Cotter RJ, Zhang Y, Wolberger C (2009) Side chain specificity of ADP-ribosylation by a sirtuin. FEBS J 276(23):7159-7176. https://doi.org/10.1111/j.1742-4658.2009.07427.x

34. Mashimo M, Kato J, Moss J (2014) Structure and function of the ARH family of ADP-ribose-acceptor hydrolases. DNA Repair 0:8894

35. Krishnan J, Danzer C, Simka T, Ukropec J, Walter KM, Kumpf S, Mirtschink P, Ukropcova B, Gasperikova D, Pedrazzini T, Krek W (2012) Dietary obesity-associated Hiflalpha activation in adipocytes restricts fatty acid oxidation and energy expenditure via suppression of the Sirt2-NAD+ system. Genes Dev 26(3):259-270. https://doi.org/ 10.1101/gad.180406.111

36. Seo KS, Park JH, Heo JY, Jing K, Han J, Min KN, Kim C, Koh GY, Lim K, Kang GY, Uee Lee J, Yim YH, Shong M, Kwak TH, Kweon GR (2015) SIRT2 regulates tumour hypoxia response by promoting HIF-1alpha hydroxylation. Oncogene 34(11):1354-1362. https:// doi.org/10.1038/onc.2014.76

37. Mock BH, Brown-Proctor C, Green MA, Steele B, Glick-Wilson BE, Zheng Q-H (2011) An automated SPE-based high-yield synthesis of $\left[{ }^{11} \mathrm{C}\right]$ acetate and $\left[{ }^{11} \mathrm{C}\right]$ palmitate: no liquid-liquid extraction, solvent evaporation or distillation required. Nucl Med Biol 38(8):1135-1142. https://doi.org/10.1016/j.nucmedbio.2011.05.007

38. Runkle AC, Shao X, Tluczek LJ, Henderson BD, Hockley BG, Scott PJ (2011) Automated production of $\left[{ }^{11} \mathrm{C}\right]$ acetate and $\left[{ }^{11} \mathrm{C}\right]$ palmitate using a modified GE Tracerlab FX(C-Pro). Appl Rad Isotopes 69(4):691-698. https://doi.org/10.1016/j.apradiso.2011.01.002

39. Tipre DN, Zoghbi SS, Liow JS, Green MV, Seidel J, Ichise M, Innis RB, Pike VW (2006) PET imaging of brain 5-HT1A receptors in rat in vivo with 18F-FCWAY and improvement by successful inhibition of radioligand defluorination with miconazole. J Nucl Med 47(2):345-353

40. Matsushima S, Sadoshima J (2015) The role of sirtuins in cardiac disease. Am J Physiol-Heart C 309(9):H1375-H1389. https://doi.org/ 10.1152/ajpheart.00053.2015

41. Houtkooper RH, Pirinen E, Auwerx J (2012) Sirtuins as regulators of metabolism and healthspan. Nat Rev Mol Cell Biol 13(4):225-238. https://doi.org/10.1038/nrm3293 